\title{
THEORETICAL STUDIES IN
}

NUCLEAR REACTION AND NUCLEAR STRUCTURE

Progress Report

J. J. Griffin and M. K. Banerjee

March 1, 1976 - August 31, 1977

\section{UNIVERSITY OF MARYLAND \\ DEPARTMENT OF PHYSICS AND ASTRONOMY COLLEGE PARK, MARYLAND}




\section{DISCLAIMER}

This report was prepared as an account of work sponsored by an agency of the United States Government. Neither the United States Government nor any agency Thereof, nor any of their employees, makes any warranty, express or implied, or assumes any legal liability or responsibility for the accuracy, completeness, or usefulness of any information, apparatus, product, or process disclosed, or represents that its use would not infringe privately owned rights. Reference herein to any specific commercial product, process, or service by trade name, trademark, manufacturer, or otherwise does not necessarily constitute or imply its endorsement, recommendation, or favoring by the United States Government or any agency thereof. The views and opinions of authors expressed herein do not necessarily state or reflect those of the United States Government or any agency thereof. 


\section{DISCLAIMER}

Portions of this document may be illegible in electronic image products. Images are produced from the best available original document. 


\title{
ORO-5126-25
}

THEORETICAL STUDIES IN

NUCLEAR REACTION AND NUCLEAR STRUCTURE

\section{Progress Report}

J. J. Griffin and M. K. Banerjee:

\author{
University of Maryland \\ Department of Physics and Astronomy \\ College Park, Maryland
}

March. 1, 1976 - August 31, 1977

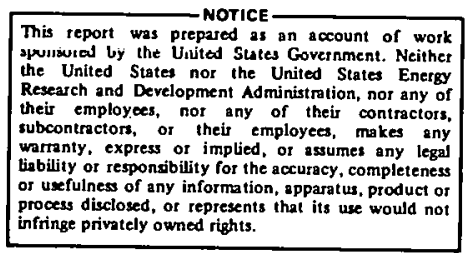

Prepared for the U. S. Encrgy Reesarch \& Development Administration under Contract \#EY-76-S-05-5126. 


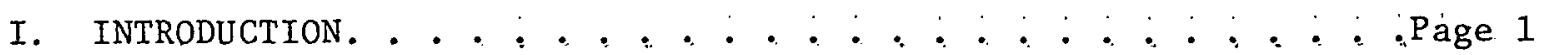

II. MANY-BODY AND FEW-BODY REACTION THEORY... . . . : : : : : :Page 2

A. Conditions for the Validity of the Lippmann Identity. : : Page 2

B. Test of Truncation in a Four-Body Problem. : : : : : Page 3

C. Studies of Trncations of Many-Body Reaction Theoreis. . . Page 4

D. Convergence of the DWBA. . . . . . . . . . . . Page 5

E. Four Body Model of ${ }^{3} \mathrm{He}-{ }^{4} \mathrm{He}$ Scattering and Reactions. . . Page 7

F. The Four Nucleon Problem. . . . . . . . . . . . . . .Page 7

G. Study of the Born-Oppenheimer Approximation Using a 3-Body Mode1................... . . Page 8

III. INTERMEDIATE ENERGY REACTIONS, OPTICALS. : . . . : : . Page 10

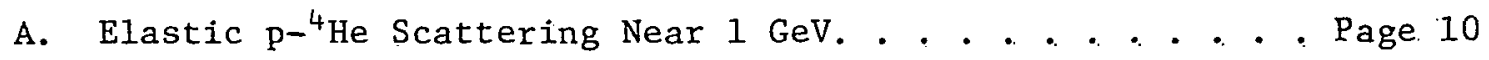

B. Exact and Approximate Multiple Diffraction Calculations, . Page 11

C. Analysis of Elastic and Inelastic $\alpha-{ }^{12}$ Data $\left(E_{\alpha}=1.37 \mathrm{GeV}\right.$ with a Multiple Diffraction Model. . . ........ Page 11

D. Relativistic Eikonal Expansion. . . . . . . . . . . . Page 12

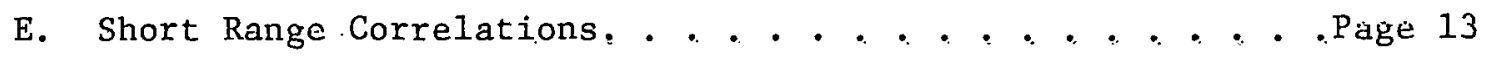

F. $\Delta$ Virtual Production Process. . . . . . . . . . . . . Page 14

G. Quasi-Free Delta Production. . . . . . . . . . . Page 15

H. Three-Body Models of the Deuteron-Nucleus System. . . . Page 16

I. Multiple Scattering Corrections to the Optical Potential: .Page 18

IV. PION REACTIONS. ...................... . . . . . . . . . . 19

A. Pion Nucleon Scattering. . . . . . . . . . . . . Page 19

B. Pion Nucleon Sigma Commutator. . . . . . . . . . . Page 20

C. Pion Production in Nucleon-Nucleon Collisions. . . . . . Page 20

D. Nucleon Knockout by Kaons. . . . . . . . . . . . . Page 21

E. Quasifree Scattering of Kaons. . . . . . . . . . . . Page 22 
F. Nuclear Momentum Distributions Probed by Quași-Free

Scattering........................ Page 23

V. NUCLEAR DYNAMTCS. . . . . . . . . . . . . . . . . . Page 24

A. Lifetime of a Determinant in Time Dependent. Hartree-Fock

Model for Nuclear Heavy Reactions. . . . . . . . . . . .Page 24

B. The Reaction Theoretical Basis for Interpreting TDHF

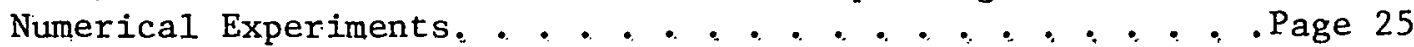

C. Theoretical Commensurability in Complex Nuclear Theories, Page 26

D. Survey of Theoretical Approaches to Heavy Ion Physics. . . Page 26

E. Rayleigh Instability in the Colliding Water Droplets. . . Page 28

F. TDHF Solution as a Dynamical Basis. . . . . . . . . Page 28

G. Instant Particle Emission in Heavy Ion Collisions. : . . .Page 28

H. Application of the. Window Formula to the Grazing Heavy-Ion

Collisions. . . . . . . . . . . . . . . . . . . . . Page 29

I. Semiclassical Approximation to the Time-Dependent Haxtree-..

Fock Theory ...................... Page 29

J. Semiclassical Approximations for a Momentum.

Dependent One-Body. Potential. . . . . . . . . Page 30

K. Shells and Clusters in Deformed Nuclei. . . . . . . . Page 31

L. Alpha-Particle Model Calculation in ${ }^{32} \mathrm{~S}$ as $\alpha$ Microscopic

Model for Fission. . . . . . . . . . . . . . . . Page 32

M. Energy Dissipation in the Fission of ${ }^{252} \mathrm{Cf}(\mathrm{sf}) . . . .$. . Page 32

N. Predictions of Ternary Fission Rates for Element 126. . . Page 33

0. An Algorithm for Non-Smoothing Contour Representations of

Two-Dimensional Arrays. . . . . . . . . . . . . . Page 34

VI. APPENDIX. . . . . . . . . . . . . . . . Page 34

4. Reeearch Puhlifations - March 1, 1976 - August 31, 1977. . .Page 35

B. Contributed Papers - March 1, 1976 - August 31, 1977. . . .Page 38

C. Invited Talks - March 1, 1976 - August 31, 1977. . . . . .Page 41

D. Papers in Preparation. . . . . . . . . . . . . . . Page 42 


\section{INTRODUCTION}

This progress report is divided into five sections covering Many-Body and Few-Body Reaction Theory, Intermediate Energy Reactions, Pion Reactions, and Nuclear Dynamics describing recent progress on thirty-five specific topics. In addition, Appendices list the publications (44), invited (13) and contributed (36) papers of the research group since March, 1976.

The long-standing interests of the Nuclear Theory Faculty are well reflected by these recent activities. Especially in the areas of eikonal descriptions of energetic reactions, where a long-standing discrepancy between Wallace's best calculation and the best available data has been resolved by a re-measurement, and in the pion-nucleus problem where the systematic development of the Chew-Low theory has been developed so as to narrow the phenomenological freedom in this area, and, incidentally, to improve the agreement with experiment.

During 1976-77, one S.W.U.M. (Seminar Workshop at the University of Maryland) meeting was held;

Pion Nucleus Scattering--The S,W.U.M. focussed on pion nucleus elastic scattering. All aspects of the problem starting from basic pion nucleon interaction going on to Pauli effect and multiple scattering were covered. Effects related to pionic field in a nucleus were also discussed. About fifty people from all parts of the country attended the S.W.U.M. University of Maryland Center for Theoretical Physics provided the expenses for T. Ericson of CERN.

Also twenty-six intermediate and long-term visitors joined the group for collaborative research for periods from one week to one year. 


\section{MANY BODY AND FEW BODY REACTION THEORY}

\section{A. Conditions for the Validity of the Lippmann Identity}

The Lippmann identity, $G_{a} G_{b}^{-1} \phi_{b}=\delta_{a b} \phi_{b}$, has been used for many years and in many-contexts by nuclear and many-body reaction theorists. It is easy to show that while the identity is true, it cannot be used in a cavalier manner. The operator $G_{b}^{-1}$ when applied to $\phi_{b}$, the on-shell channel state, gives $O$ (or +7 if the small imaginary part of the energy is properly maintained). The point of the identity is that only the Green function of the same channel has the appropriate singularity to cancel the zero of $G_{b}{ }^{-1}$.

Care is needed when the identity is used in a reaction theory as singular operators may be applied to $G_{a} G_{b}^{-1}$ from the left. Even if the $G_{a}$ doesn't have the singularity necessary to cancel the zero, the singular operator might, thereby invalidating the identity.

We have derived the conditions on the use of this identity. We find that the identity holds in the form

$$
1 \text { im } A(n) G_{a}(E+i \eta) G_{b}^{-1}(E+i n) \phi_{b}=A(0) \delta_{a b} \phi_{b}
$$

only if the perturbation expansion of the operator A consists of strings all of which have a potential external to the partition $b$ before it has an infinite string of potentials internal to $\mathrm{b}$ arranged in all orders (when considered as strings beginning on the right).

This theorem has been applied to the off-shell transformation which yields the Bencze-Redish-Sloan Many-Body equations to demonstrate the correctness of the transformation. (E. F. Redish, P. C.Tandy, Australian National University, Canberra, \& M. L'Huillier*)

\footnotetext{
* Visitor during 1975 from I.P.N., Orsay France
} 


\section{B. Test of Truncation in a Four-Body Problem}

The most important property determining the value of a many-body reaction theory is the accuracy of its truncations. In general, no exact calculation can be expected for a quantum mechanical problem with more than 5 particles, so if a many-body reaction theory is to have any validity, it must have truncations, i.e., severe approximate treatments, which retain most of the important processes of the theory and which give reasonably accurate results.

On conjecture which is common in describing nuclear reactions is that intermediate states with more than 3 clusters are either not important or can be treated statistically. Different reaction theories differ in how the dynamics is distributed through different intermediate states so various theories which can be expected to give the same answer if treated exactly may give very different answers when treated approximately.

We have studied the accuracy of truncations in a four-body model. This four-body model is based on a field theory and is equivalent to the GrassbergerSandhas four-body equations when pole approximations are made. The intermediate. states in this model include two, three, and four body states. The calculation was carried out exactly and in approximations in which four and three body intermediate states were successively thrown away. We find that in this formulation of the many-body equations, the only states which can be dropped are four-body intermediate states in the deuteron-deuteron channel. The four-body intermediate. states occurring in "vertex renormalizations," i.e., while a particle or cluster is being transferred, must be retained. The three-body intermediate states retain their importance even when the threshold for these processes are pushed up well above the energy considered. Etudics are in progress to detcrmine whether this lack of truncatability arises from the particular form of the equations or is a general feature of 
few-body equations. (E. F. Redish and Antonio C. Fonseca)

\section{Studies of Truncations of Many-Body Reaction Theories}

The many-body reaction theories of Bencze-Redish-Sloan (BRS), KouriLevin-Tobocman (KLT), and Grassberger-Sandhas-Yakubovskii (GSY) have been studied in truncation.

The GSY theory has equations with a very complicated index structure and no truncations for many-body problems are known. These equations are only considered for the case $\mathrm{N}=3$ and 4 . The KLT equations are considered in two forms; the complete equations on all two-cluster channels are shown to have very complicated truncations which are not easily treatable, and the partial equations on a particular subset of channels have simple truncations bur are not easily correctable. Truncations of the BRS equations are both possible and correctable.

Natural truncations of the three sets of equations to two-cluster channels are considered from the point of view of time reversal invariance (TRI) and unitarity. We show that only the truncated GSY equations satisfy TRI. The discrepancy in the other two sets of equations arises from the well-known post-prior ambiguity of the DWBA and is expected to be sma11. All three sets of equations satisfy a truncared form of unilarily. Limiting cases are also being considered. We are looking at taking the limit of the three theories for the case of a weak probe (termed an "electron") scattering from a nucleus in the limit that the electron-nucleon interaction goes to zero. In this case, the natural truncations should lead to the Born approximation. A second example is. a strongly interacting probe with a very short range interaction (termed a "Kaon"). scattering from a nucleus in the limit that the nucleus becomes very dilute. In this case the natural truncations should lead to a multiple scattering series beginning with an impulse 
approximation. It has already been demonstrated that in some of the cases these results are not obtained with the "obvious" truncations. Further investigations are in progress. (E. F. Redish and R. Dixon)

D. Convergence of the DWBA

The Distorted Wave Born Approximation (DWBA) is used extensively in nuclear direct reaction theory for the analysis of inelastic scattering and transfer reactions. Often, corrections to this treatment are required and are usually made by the calcuations of multi-step corrections. The criteria governing the convergence of this procedure is poorly understoody. We have therefore undertaken a model study to consider this problem.

We have investigated inelastic scattering of a nucleon from a nucleus with two and three states. The form factors and direct matrix elements are taken to be separable. These models are then solvable analytically if the separable potentials are taken to have Yamaguchi form. Only Suwaves are considered. A distorting potential is added and subtracted to the free Hamiltonian and an integral equation constructed. The inhomogeneous term of the equation is the DWBA and the iteration of the equation leads to a multi-step DWBA series. The convergence of the series is determined by the magnitude of the largest eigenvalue of the kernel of this equation. This is the "small parameter" which governs the convergence of the DWBA series. This parameter is considered as a function of the parameters of the problem (energies, strengths, ranges) written in dimensionless form. The Convergence parameter is calculated for a range of the dimensionless parameters and for a number of different choices of the distorting potential.

Three distorting potentials are considered: (1) the folding potential (i.e., the diagonal matrix elements of the true Hamiltonian), (2) the optical 
potential (i.e., that potential which gives the elastic scattering exactly), and (3) the "ideal" distorting potential (i.e., that potential which minimizes the convergence parameter). The optical potential can be found exactly in both the two and three channel models, while the ideal potential has only been determined in the two channel case.

We find that in the two channel model the convergence of the series is limited largely to the region where the coupling strength is less than the diagonal potential strength. The folding model almost always produces a more convergent series than the truc optical potential. The ideal potential yields a convergent series everywhere, but in the regions where the other distortions lead to divergent series, the ideal potential is only barely convergent (convergence parameter on the order of 1). The failure of the optical potential to provide a better DWBA than the folding potential is quite surprising. One possibility considered was that this two channel model did not satisfy the conditions that are supposed to make the DWBA work, namely, when inelastic scattering occurs, in most cases it is to a channel different from the one considered. The likelihood of returning from any other channel to the specific inelastic channel observed is small. In our two channel model the only inelsatic scattering that takes place is to the observed channel. To remedy this defect we considered a three channel model. In this model a strong coupling was assumed from the elastic to the third (absorbed). channel but no coupling between the second (observed) channel and the absorbed channel. This mocks up very well the situation that should make the DWBA work. We find in this model that the folding potential still produces better convergence than the optical potential. We must conclude that the accuracy of the standard DWBA depends on considerations other than absorption, such as the geometrical dominance of surface regions. Further investigations of these results are in progress. (E. F. Redish and Dan MacMillan) 
E. Foux Body Model of ${ }^{3} \mathrm{He}-{ }^{4} \mathrm{He}$ Scattering and Reactions.

Since the alpha particle is tightly bound, at low energies it should be treatable as an elementary particle. Few-body cluster-cluster scatterint involving one or more alpha particles may then be treated in few body models. The reactions induced by ${ }^{3} \mathrm{He}$ incident on alpha particles is considered in a four body model consisting of three nucleons and an alpha. The model chosen is field theoretic and includes the vertices $n+n-d, p+d-$, $\mathrm{p}+\therefore{ }^{5} \mathrm{He}, \mathrm{d}+-{ }^{6} \mathrm{Li}$, and $\mathrm{N}^{5}{ }^{5} \mathrm{He}-{ }^{6} \mathrm{Li}$. The three variable four body equations reduce in this model to one variable equations which can be solved by contour rotation. Three and four body intermediate states are treated by pole approximations and iteration. Since the model sums all the graphs coming from a model field-theoretic Hamiltonian, the results are guaranteed to be unitary including three and four body breakup.

The calculation has been done with $S$ wave interactions, the nucleonalpha $P$ wave being approximated by an S-wave separable potential with. a barrier. The form factor is adjusted to give the right threshold behavior, but the angular momentum coupling is not correct. Reasonably. good cross sections are obtained for elastic and transfer scattering, considering the zero parameter nature of the calculation. The magnitudes are excellent but the angular distributions don't have the experimentally observed structure. This should be improved by including the correct angular momentum coupling. (Antonio C. Fonseca)

\section{F. The Four Nucleon Problem}

The four nucleon problem is solved in a field theoretic model. The nucleon is taken to have both spin and isospin $1 / 2$ and interact to:form either a deuteron or anti-bound deuteron quasiparticle state. In the threebody sector the amplitudes are assumed dominated by the $=0$ states and three quasi-particles are introduced to approximate the three-body scattering in these states. 
In the four body sector after partial wave decomposition a set of coupled one dimensional integral equations are obtained for the processes $\mathrm{n}+{ }^{3} \mathrm{He}-\mathrm{n}+{ }^{3} \mathrm{He}, \mathrm{p}+{ }^{3} \mathrm{H}$, and $\mathrm{d}+\mathrm{d}$ as well as for the $\mathrm{d}+\mathrm{d}$ initiated processes. These equations were solved by matrix inversion on a rotated contour. Total reaction cross sections (including breakup) were obtained from unitarity. These cross sections fit the data extremely well up to energies of about $40 \mathrm{MeV}(\mathrm{lab})$. The angular distributions for the elastic and transfer reactions are also quite good. (Antonio C. Fonseca)

\section{G. Study of the Born-Oppenheimer Approximation Using a 3-Body Model}

The Born-Oppenheimer approximation for a description of molecular states is tested in a three-body model consisting of two heavy particles and a light. The heavy particles interact with the light through a separable Swave potential. The two heavies do not interact with each other directly. Contrary to most experience in the few-body problem with equal mass particles, we find that as the ratio of the heavy particle's mass to the light particle's mass, $R$, increases, one finds an increasing number of bound states and resonances in many partial waves. For $\mathrm{R}=$ infinity, the number of three-body bound states becomes infinite and has an accumulation point at the two-body threshold. For $\mathrm{R}$ rcasonably large a clcar vibration-rotation structure is seen and the level structure resembles that of a three-dimensional anharmonic oscillator.

The same three-body problem was then solved with the Born-Oppenheimer (BO) approximation. The results obtained for the binding energy were compared with those given in the exact 3-body calculation. For $\mathrm{R}>30$ the results agree to better than 3 per cent. As $R$ decreases from there the results worsen considerably. We find that the BO approximation can be improved 
considerably by using the reduced mass of the light particle with the two heavies instead of just the mass of the light particle. With this correction, the BO approximation agrees with the exact to 9 percent for $R=5$ and 12 percent for $R=1$. Such good agreement between the two methods suggests that the BO approximation may. be a very valuable starting point for three-body bound state calculations even for equal mass particles.

In the scattering region we solve the three-body equations for the various two to two processes. The angular distributions show a pronounced diffraction pattern much like heavy.ion scattering data. For identical cores, the elastic phase shifts present the usual odd-even behavior due to the exchange nature of the interaction. The even partial waves are attractive and may resonate. As $\mathrm{R}$ increases resonances appear at higher and higher values. The odd partial waves are repulsive and are the only ones to contain breakup inelasticity. The suppression of the inelasticity in the even partial waves seems to be a genuine three-body effect that we have not yet fully understood. Further studies are planned. (Antonio C. Fonseca and P. Shanley, U. of Notre Dame, Indiana) 
III. INTERMEDIATE ENERGY REACTIONS, OPTICAL MODELS

A. Elastic $\mathrm{p}^{4} \mathrm{He}$ Scattering Near $1 \mathrm{GeV}$

In the past year, two new experiments on $1 \mathrm{GeV} \mathrm{p-} \mathrm{He} \mathrm{elastic} \mathrm{scattering}{ }^{1}, 2$ have been performed which provide data on both the differential cross section and polarization. These new data have been very successfully explained using multiple diffraction theory and one of the principal conclusions of our analysis is that the recently normalized Saclay $\mathrm{p}-{ }^{4} \mathrm{He}$ data at $1 \mathrm{GeV}^{3}$ appear to be incorrect by about $60 \%$ in normalization.

The multiple diffraction theory calculations for $\mathrm{p}-{ }^{4} \mathrm{He}$ are by far the most complete analysis to date. The leading order in $\mathrm{k}^{-1}(\mathrm{kh}=\mathrm{cm}$ momentum). corrections to Glauber theory, as determined by the high energy expansion method $^{4}$, have been incorporated. These corrections are shown to be rather small at forward angles thereby motivating a close check of theory and experiment. The spin and isospin dependence of the nucleon-nucleon amplitudes has been incorporated and the ${ }^{4} \mathrm{He}$ wave functions used fully incorporate the center-of-mass constraint while providing very good reproduction of the ${ }^{4} \mathrm{He}$ charge form factor and coordinate space charge density.

1. GeV proton-scattering analyses are based on the existing phaseshift amplitudes for proton-proton ( $p p)$ scattering. However, the proton-neutron (pn) spin-flip amplitude, $\mathrm{C}_{\mathrm{pn}}$, is essentially undetermined at this energy. The new $1 \mathrm{GeV} \mathrm{p}-{ }^{4} \mathrm{He}$ polarization data therefore provide an invaluable constraint on the pn amplitudes and our analyses are based on a $\mathrm{C}_{\mathrm{pn}}$ amplitude which fits both the known pn and the new $\mathrm{p}-{ }^{4}$ He polarization measurements. Nevertheless, all of the above refinements fail to explain the absence of a deep diffraction minimum in the $\mathrm{p}-{ }^{4} \mathrm{He}$ data.

1. J. V. Geoga, et...al. Phys. Rev. Lett: 38, 1265 (1977).

2. R. K1.em, et. a1. Phyc: Rcv. Lctt: 38, 1272(1977).

3. E. Aslanides, et. a1. Phys. Lett. 68B, 221(1977).

4. S. Wallace, Phys, Rev. C12, $179(1.975)$. 


\section{B. Exact and Approximate Multiple Diffraction Calculations}

One of the issues which is raised by large angle scattering experiments on light nuclei (e.g. D, ${ }^{3} \mathrm{He},{ }^{4} \mathrm{He}$ ) is the adequacy of multiple scattering theory at large angles. In an attempt to clarify the adequacy of eikonal approximation (Glauber Theory), the high-energy expansion method of Wallace and the noneikonal approach of Gurvitz, Alexander and Rinat ${ }^{5}$, calculations were made for an exactly soluble model of nucleon-deuteron scattering. Sparrow's exact fixed scatterer model calculations were used as a benchmark against which the approximate calculations were compared. Although: the fixed scatterer model omits some physically important effects, the results showed the general adequacy of the high-energy expansion and noneikonal calculations to angels of $80^{\circ}$ in the $\mathrm{cm}$. At forward angles the eikonal expansion method reliably gives the overlapping potential corrections to Glauber theory.

A very interesting possibility for backscattering by very light nuclei, namely the predominance of single scattering proposed by Gurvitz et. al., was also explored in the fixed scatterer model calculations. When the single scattering, nucleon-exhange, process dominated, the error made in approximate calculations was overwhelmed at back angles. In this case, approximate descriptions, of the multiple scattering terms (Glauber, etc.) were adequate to backscattering angles vor very light targets: (Y. Alexander, S. J. Wallace, and D. Sparrow)

C. Analysis of Elastic and Inelastic $\alpha-12^{\prime \prime}$ Data $\left(E_{\alpha}=1.37 \mathrm{GeV}\right)$ with a Multiple: Diffráćtion : Módel

High energy $\left(E_{\alpha}=1.37 \mathrm{GeV} \alpha-{ }^{12} \mathrm{C}\right.$ distributions were analyzed with an eikonalized distorted wave impulse approximation. Using an elastic phase

5. S. Gurvitz, Y. Alexander and A. Rinat, Ann. of Phys. 93, 152(1975);98,346(1976). 
function parametrizal to fit the elastic $\alpha-{ }^{12} \mathrm{C}$ distribution, and inelastic form factcrs from electron scattering data, we obtained reasonable agreements for the $2^{+}$and $0^{+}$distributions. However, there was a definite disagreement for parts of the $3^{-}$distribution where a predicted minimum and subsequent maximum are absent. Essentially the same measure of agreement and disagreement has been observed for the $\mathrm{p}^{12} \mathrm{C}$ data at $1.05 \mathrm{GeV}$. On the basis of this and previous work, it seems that hadronic projectiles excite natural parity levels through a transition density which is closely related to transition change form factors, with initial and final state distortions described by elastic data. The $3^{-}$anomoly may be due to effects such as virtual isolar production or noneikonal effects in multiple scattering. (Y. Alexander and A. Rinat, Weizman Institute of Science, Rehovot, Israel).

\section{Relativistic Eikonal Expanision}

The nature of high energy scattering within relativistic quantum field theory has been greatly clarified by the use of the eikonal approximation in the last 10 years. Following the pioneering work of Cheng and $\mathrm{Wu}^{6}$, Abarbanel and Itzykson ${ }^{7}$ showed that the high energy limit extracted from an infinite set of Feynman diagrams ("generalized ladders" set) for 2-particle scattering could be obtianed in a simple and transparent fashion using the relativistic eikonal approximation.

A recently completed manuscript develops, through second order, a relativistic eikonal expansion for scalar scattering which takes as its zeroth term the relativistic eikonal approximation. The results show a remarkable correspondence between the fully-relativistic high-energy

6. H. Cheng and T. T. Wu, Phys. Rev. Lett: 22, 666(1969); Phys. Rev: 186,1611(1969).

7. H. D. I. Abarbanel and C. Itzykson, Phys. Rev. Lelt: 23, 53(1969), 
scattering amplitude and the results: of potential theory. In large part the relativistic results can be obtained from a Schrödinger-like wave equation with a local potential $U(r)$, provided the reduced mass $m_{i} m_{2} /\left(m_{1}+m_{2}\right)$. is replaced by $E=E_{1} m_{2}\left[m_{1}{ }^{2}+m_{2}^{2}+2 m_{2} E_{1}\right]^{-1 / 2}$, where $E_{1}$ is the $1 a b$ energy of particle 1 (the projectile) and $m_{1}, m_{2}$ are the masses of particles 1 and 2 . This relativistic kinematics prescription has already been found very useful in formulating a high energy expansion for nuclear multiple scattering ${ }^{4}, 8$.

At a more basic level, the relativistic eikonal expansion provides a clear insight into the role of the relative time coordinate in relativistic scattering (it enters into the 2 nd order corrections to the eikonal limit). Our results are intended to provide a valid high energy expansion of a fundamental scattering model (the generalized ladders set of Feynman diagrams ( which we believe provides a benchmark against which one can test the validity of various approximate kernels to be used in conjunction with relativistic wave equations of the Bethe-Salpeter or Sugar-Blankenbëcler types. (S. J. Wa1lace and J. McNeil)

\section{E. Short Range Correlations}

Work near completion extends the analysis of $1 \mathrm{GeV} \mathrm{p}-{ }^{4}$ He scattering to. larger momentum transfer, $-t \simeq 1.2(\mathrm{GeV} / \mathrm{c})^{2}$, and investigates the role of short range correlations (SRC). Using Jastrow-type wave functions with several correlation. lengths and calculating through fourth order multiple scattering, our results show very minor effects due to SRC. In each case the wave function parameters are chosen to reproduce the ${ }^{4}$ He form factor with cm correlations: Even will belter knowledge of pn amplitudes, there is little hope that one can extract SRC information from the $p-{ }^{4} \mathrm{He}$ elastic

8. S. K. Young and C. W. Wong, Phys. Rev. C15, 2146(1977). 
scattering.

The results of this analysis show much less sensitivity to the correlations than the optical potential work of Lambert and Feshbach ${ }^{9}$. The reason for this lies in the treatment of center-of-mass correlations which. dominate the correlation effect for a nucleus as 1 ight as ${ }^{4} \mathrm{He}$. In the multiple diffraction approach, the cm constraint is kept to all orders while in the optical potential approach, one expands the two-body density in the fashion

$$
\rho(2)\left(\vec{r}_{1}, \vec{r}_{2}\right)=\rho\left(\vec{r}_{1}\right) \rho\left(\vec{r}_{2}\right)\left[C\left(|| \vec{r}_{1}-\vec{r}_{2} \mid\right)+i 1\right]
$$

center-of-mass correlation effects essentially invalidate this model of the two-body density for ${ }^{4} \mathrm{He}$ for Jastrow-type wave-functions. (Y. Alexander and S. J. Wallace)

\section{F. $\quad$ : Virtual Production:Process}

he single most important refinement of Glauber theory which is needed to explain the data is the $\Delta$ - virtual production process in which the fast proton becomes excited to a $\Delta$ isobar in one scattering and de-excited back to a proton in a second scattering, by a different target nucleon. Because the $\mathrm{pp} \rightarrow \mathrm{N} \Delta$ cross section is about $20 \mathrm{mb}$ at $1 \mathrm{GeV}$, this process can be quile important, although spin and 1sospin consideratione have led to some doubts.

A spin- and isospin dependent model of the $\mathrm{pp} \rightarrow \mathrm{N} \Delta$ amplitude has been developed and the extra multiple scattering amplitudes where a $\Delta$ is in the intermediate state have been calculated. Even with the spin and isospin effects properly accounted, the $\Delta$ - virtual production process provides substantial filling of the ${ }^{4}$ He first diffraction minimum. Multiple

9. E. Lambert and H. Feslibach, Ann. of Phys. (N.Y.) 76, 80(1973). 
diffraction calculations with the $\Delta$ process included provide excellent fits to both the differential cross section and polarization data, whereas without the $\Delta$ process it does not appear possible to fit the data using reasonable pp and pn amplitudes. (S. J. Wallace and Y. Alexander)

\section{G. Quasi-Free Delta Production}

When the total reaction cross-section for protons on light nuclei at energies of a few hundred $\mathrm{MeV}$ is considered, one finds that almost all the. cross-section is accounted for by single nulceon knockout via a quasi-free scattering mechanism. If one considers the $1 \mathrm{GeV}$ data, however, one $f$ inds that only about $1 / 3$ of the total reaction cross-section is provided by this process.

In order to try to account for the missing cross-section we have considered the fact that at $1 \mathrm{GeV}$ quasi-free scattering can produce pions. We have calculated this quasi-free reaction process using a model of nucleonnucleon inelastic scattering which describes the reaction $N+N \rightarrow N+\Delta$. In free nucleon-nucleon scattering this process has a cross section approximately equal in magnitude to the elastic scattering.

When this amplitude is inserted into a PWIA calculation of the reaction $\left(\mathrm{P}, \mathrm{p}^{\prime}\right)$ we find the standard quasi-free peak at an energy loss of $100-200$ $\mathrm{MeV}$ and a second peak at an energy loss of about $400-500 \mathrm{MeV}$. This second peak corresponds to the production of an (unobserved) delta. The total cross section to this second peak is about twice the calculated quasi-free crosssection. The reason for the enhancement compared to the free cross sections is that essentially all the knockout and quasi-free reaction comes from a momentum matched scattering (momentum transfer $=0$ ). The relevant comparison is therefore not the total elastic vs. the total free reaction cross section, but rather the ratio of the forward cross-stctions. 


\section{H. Three-Body Models of the Deuteron-Nücleus System}

There is strong evidence from the study of $(p, d)$ and $(d, p)$ reactions on a variety of heavy targets that three-body intermediate states play an important role in these processes. A recent example is provided by the $65 \mathrm{MeV}$ (p,d) data obtained at Maryland on targets of ${ }^{12} \mathrm{C},{ }^{14} \mathrm{~N},{ }^{16} \mathrm{O},{ }^{40} \mathrm{Ca}$, and ${ }^{90} \mathrm{Zr}$. It has been shown that the standard DWBA which, roughly speaking treats the (p,d) reaction as a pair of two-body channels coupled perturbatively, does not give an adequate description of the observed angular distribution of the outgoing deuterons. The inclusion of contributions from certain three-body channels (neutron and proton in an unbound state, plus residual nucleus) using the adiabatic prescription proposed by Johnson and Soper gives an excellent fit to the data. It was shown by Johnson and Soper that a similar treatment of the contributions of these channels to elastic deuteron scattering works quite well, and subsequent work by Austern, Johnson and Tandy, Rawitscher and others has thrown considerable light on the nature of the approximation involved. However, none of these approaches had provided a basis for a systematic theoretical understanding of the role of three-body channels as a function of energy, mass number of target, and Qvalue. This kind of understanding is crucial as the need for generalization to nuclear reactions of a more complex nature is being developed. With these. considerations in mind research has been pursued along the lines outlined below.

\section{Validity of the Adiabatic Prescription as an Approximation to the Three-body Problem}

The role of three-body effect in $(p, d)$ reactions has been re-examined within the framework of the adiabatic approximation of Johnson and Soper and the results applied to Maryland data on light nuclei at $65 \mathrm{MeV}$. Excellent agreement with experiment has been obtained.

This work. was submitted to the APS Meeting, Washington, D. C. April, 1976 and is in process of publication in Nuclear Physics (R. C. Johnson) 


\section{Development of a Correct and Useful Three-Body Hamiltonian}

In the course of the study of work by Redish and Kouri and Levin with the aims of this project in view it has become clear that many of the truncations of the many-body problem suggested by these authors do not satisfy the fundamental requirements of reciprocity. A paper outlining some consequences of this and possible ways of developing formalisms which do not have this property is in the course of preparation in collaboration with Redish and Viscar. (R. C. Johnson)

\section{Spin Dependent Effects in Reactions Involving Deuterons}

As part of this research effort a course of lectures on polarization effects in nuclear reactions was prepared and delivered. The lecutre notes are currently being prepared for circulation. An invited talk on this subject was given at the APS Meeting, Washington, D. C., April, 1976.

A novel method of partial wave analysis for spin-s particles has been developed which has some advantage when spin-dependent forces of rank higher than 1 are important. The extent to which observable effects die to second rank tensor forces can be separated out in the high angular momentum limit has been discussed. This approach may be useful in the. study of high energy nucleon-nucleon scattering. This work has been written up and submitted to Nuclear Physics.

Considerable effort has been put into understanding the validity and limitations of the approach of Ioanides and Johnson to the calculation of Pauli Exclusion Principle and tensor force effects in deuteron scattering. These results will be prepared for publication in the next severa1 months.

The study of the ole of ${ }^{\perp} s$ states in $(d, p)$ reactions near the 
Coulomb barrier is continuing. It has become clear that a more accurate treatment of the energy difference between elastic and break-up channels will be necessary and the associated modification of the formalism are underway in collaboration with. G. L. Wales at Surrey. Considerable progress has been made on the study of Coulomb polarizability and numerical results should be available in the near future. (R, C. Johnson)

\section{Multiple Scattering Corrections to the Optical Potential}

A study has been completed which throws an interesting light on the validity of the closure approximation and the role of quasi-free scattering in the multiple scattering theory of Watson. This work is being prepared for publication.

A new phenomenological approach to nuclear scattering by nuclei in the $50-" / 50 \mathrm{MeV}$ region which incorporates some of the non-1ocal and $\ell$-dependent features of multiple scattering effects is being developed in collaboration with L. W. Woo.

The relationship between the prediction of Jackson, Johnson and others for folding models for the a-nucleus optical potential and conventional phenomenological models is being studied in collaboration with N. S. Wall and A. A. Cowley. $\Lambda$ paper is being prepared for publication. (R. C. Johnson) 


\section{PION REACTIONS}

\section{A. Pion Nucleon Scattering}

We proposed a theory of low energy pion nucleon scattering which may be viewed as a logical extension of the classic work of Chew and Low. The basic dynamical imputs were (1) PCAC, i:e., the pion field, is proportional to the divergence of the axial vector current, and (2) absence of a $\rho$ meson term in the interaction Lagrangian. The last assumption does not exclude a $\rho$ meson. The latter is interpreted as an interacting two-pion system rather than a basic field.

Unlike the Chew-Low theory which dealt with the P-wave interaction only our theory was intended to be applicable to the S-wave interaction also. So we had to include the effect of single boson exchange between a pion and a nucleon. In the Low expansion of the scattering amplitude the single boson exchange appears as a so-called seagull term. Since we assume that there is no basic $\rho$ meson we have only an isoscalar seagull. This unknown quantity is eliminated in favor of the soft-pion limit. We can formally write down the latter because we use PCAC. The process of elimination gives a once-subtracted Low equation which is by itself a great advantage.

The resulting theory has two major parameters : $\sigma(\pi N)$, the sigmacomputator, and $\overline{\mathrm{g}}_{\pi}=\left|\mathrm{g}_{\pi}\left(4 \mathrm{M}^{2}\right)\right| \cdot \sigma(\pi \mathrm{N})$ characterizes the main isoscalar mechanism and $\bar{g}_{\pi}$ the main isovector mechanism. We obtained very satisfactory fit with the low energy S-wave phase shifts of Carter, Bugg, and Carter with $\sigma(\pi \mathrm{N})=25.5 \mathrm{MeV}$ and $\overline{\mathrm{Q}}_{\pi}=11.7$. A short paper describing this work will appear in the September 1, 197.7 issue of Physical Review D. A Ionger paper giving all the technical detalis has been submitted to Physical Review C. (M.K, Banerjee and I,B. Cammarata)*

* 1976 and 1977 Summer Visitor from Virginia Polytechnic Institute. 


\section{B. Pion Nucleon Sigma Commutator}

The value of $\sigma(\pi N)$ obtained in the previous work is about half of what is belicved to be the correct value, often referred to as the "world value," of $65 \pm 5 \mathrm{MeV}$. We inyestigated the reason behind this discrepancy and pointed out that the large value is entirely due to errors of extrapolation. A paper on this work has been communicated to Physical Review D. M.K. Banerjee and J.B Cammarata*).

\section{Pion Production in Nuclenn-Nucleon Collisions}

We have been able to extend the theory on pion production in nucleonnucleon collision developed by Banerjee, Levinson, Shuster and Zollman (BLSZ) by including more intermediate states than was previously done. These states are pion-nucleon elastic scattering and the pion-deutron breakup channels. We are therefore able to investigate the effects of pion-nucleon resonances on pion production. We have derived an equation for the pion production amplitudes. This expression reduces exactly to the analytic form obtained by.BLSZ when only the nucleon-nucleon rescattering is taken into account. When effects of pion-nucleon elastic scattering and pion-deuteron brcakup are included, the production amplitudes can be shown to have two terms, one of which can be analytically expressed which the other much be solved numerically. (M.K. Banerjee and K.-H. Yang).

\footnotetext{
* 19.76 and 1977 Summer Visitor from Virginia Polytechnic Institute.
} 
This simple mechanism therefore seems able to account forthe missing nucleon-nucleus reaction cross-section without invoking complicated multiple scattering processes. Investigations are under way to see whether the decay of the delta will smear out the peak too much to be observed. If not, the process should be observable in current $\left(p, p^{-}\right)$experiments at Los Alamos and Argonne. Under any circumstances, the process investigated is of considerable importance in understanding the overall reaction mechanism of nucleon-nucleus scattering above the pion threshold. (E. F. Redish, Y. Alexander, and S. J. Wa1lace)

\section{Nucleon Knockout by Kaons.}

It has recently been proposed by G.E. Walker and C.B: Dover that the $\mathrm{K}^{+}$meson would provide a useful probe of nuclear momentum distributions through the $\left(\mathrm{K}^{+}, \mathrm{K}^{+} \mathrm{P}\right)$ reaction. This meson is an attractive probe since the (K_N) cross section is slowly varying in energy and arises from a very short range force $(r \sim) .25 \mathrm{fm})$. The cross section is also much smaller than a nucleon-nucleon cross-section but much larger than the electron-neutron cross section. These features give $(\mathrm{K}, \mathrm{Kp})$ an advantage over the competing reactions $(p, 2 p)$ and $(e, e p)$. The short range and slow energy variation means that the off-shell effects should be reduced compared to $(p, 2 p)$ and the small size of the cross-section means that distortion and multi-step processes should be small compared to $(p, 2 p)$. The fact that the cross-section is hadronic and not electromagnetic means that the absolute cross-sections for (K,KP) will be much larger than for (e, ep). The (e,ep) reaction also has some difficulty exciting deep hole states since the slowly varying Coulomb force does not support large energy or momentum transfers.

Thiese facts cumbine Lu suggest that the $K$ would be the best probc of 
deep nuclear hole states. We investigated this hypothesis by calculating the cross section for knockout of a ls proton from ${ }^{40} \mathrm{Ca}$ by a $300 \mathrm{MeV}$ Kaon using a DWIA and the code AAB of R.D. Koshel. A factorized amplitude was used assuming a half-shell $\mathrm{T}$ matrix approximated by a final energy prescription:. The distortions were calculated using a Kisslinger optical potential. Both the $\mathrm{T}$ matrix and the optical potential were constructed out of amplitudes in the literature. The resulting cross-sections for the coincidence experiment peaked at values of about $2 \mu \mathrm{b} / \mathrm{sr}^{2}-\mathrm{MeV}$. As expected, this is less than $(p, 2 p)$ but greater than (e,ep)... The distortion effects were suppressed and showed that the Kaon distortion only made a difference of about a factor of 2 in the absolute cross-section compared with a factor of 30 for $(p, 2 p)$. This effect should be much more amenable to an exact theoretical analysis than that of $(p, 2 p)$ and should allow the Kaon to probe the nculear interior much more satisfactorily than the proton:

The only difficulty with performing the experiment is the present inadequacy of Kaon beams. Presently, about $10^{5}$ kaons per second can be put on target, with upgradings to $10^{7}$ being discussed. We calculate that $10^{7}-10^{8}$ Kaons per second will be needed before the experiment can produce counting rates sufficient to yield statistics competitive with the (ep) experiment. (E.F. Redish, P. Moffa and R.D. Koshe1*).

\section{E. Quastlree Scallering of Kauns}

The inclusive scattering reaction $\left(\mathrm{K}^{+}, \mathrm{K}^{+}\right)$on ${ }^{12} \mathrm{C}$ was calculated in a PWIA. Momentum distributions and the reaction mechanism were checked by

* Visitor from Ohio University, Athens, Ohio. 
comparing calculations of $\left(e, e^{1}\right)$ on ${ }^{12} \cdot C^{\prime}$ with data. Our resulting prediction of $\left(\mathrm{K}^{+}, \mathrm{K}^{+}\right)$has several attractive features, primarily that the weak absorption of the $\mathrm{K}^{+}$in the nuclear matter allows distortion to be neglected. (Y. Alexander and P.J. Moffa).

F. Nuclear Momentum Distributions Probed by Quasi-Free 'Scattering

The backward inelastic scattering of protons from nuclei at intermediate energies shows a particularly simple structure when expressed in terms of the minimum momentum a nucleon must have in the nucleus in order to give the observed backward going nucleon in a one-step quasi-free scattering. This result led Amado and Woloshyn to propose a simple form for the momentum distribution of nucleons in nuclei based on arguments arising from a one-dimensional model. This proposed momentum distribution gives a reasonable fit to the backward inelastic data.

We studied the implications of using this momentum distribution at low momentum transfers, near the intermediate energy quasi-free peak. We find that a different parameter set is required to fit the low momentum transfer (quasi-free) data than is required to fit the backward inelastic data. We conclude that the $\left(p, p^{\prime}\right)$ reaction cannot be simply described at all angles by a quasi-free mechanism with a simple momentum distribution of the Amado-Woloshyn type. (E.F. Redish, Y. Alexander and N,S, Wa11). 
V. NUCLEAR DYNAMICS

\section{A. Lifetime of a Determinant in Time Dependent Hartree-Fock Model for}

Nuclear Heavy Reactions,

During this period one specific aspect of the nuclear Hartree-Fock

theory. was studied, the decay of the assumed singleideterminant into

correlated multi-determinantal components as the reaction process pro-

ceeds under the exact propagation in a Schrodinger theoretical framework. We have succeeded in formulating an uncertainty principle between the energy spread of the approximate time dependent Hartree-Fock determinantal wave function and the quantity which characterizes the "Dependency", or non-determinantness, of the wave function. This uncertainty principle allows one to infer from some knowledge of the dispersion of the energy about its value for a given determinant wave function under exact Schrbdinger propagation and time.

The results show that even for an isolated freely translating nucleus the lifetime for a determinant is not much longer than the lifetime of a nuclear heavy ion reaction. For two nuclei in contact, it is also shown that the lifetime will be even shorter, so that the assumption of a single determinantal approximation to a wave function for a time as long as a nuclear heavy ion requires to occur, is rather marginal. We have therefore suggested that numerical time dependent Hartree-Fock calculations ought to incorporate an estimate of this lifetime for the specific circumstances which are being calculated, in order to validate the relevance of the basic Hartree-Fock assumption over an adequate time to complete-the reaction description. (J:J: Griffin and P.C. Lichtner). 


\section{B. The Reaction Theoretical Basis for Interpreting TDHF Numerical Experi- ments.}

As part of the program of assessing the TDHF as a theoretical appooach to nuclear heavy ion reactions, we have focused on the question of the physical content of the Hartree-Fock description. One result has been a formulation of the reacion information which can be extracted from time dependent Hartree-Fock (i.e. single-determinantal) calculations in terms of certain " 8 "-matrix amplitudes for a transition from an ingoing channel, $i$, to an outgoing channel, f. Such an approach leads one to emphasize the importance of the appropriate labels for asymptotic states as a characteristic of the physical content of any reaction theory, and in particular for the TDHF reaction theory:

We have set the question whether the very minimal requirements on the qualitative nature of the answers which one demands from any reasonable reaction theory allows such a "\&"-matrix theory to stand as a viable mechanism method for interpreting TDHF numerical experiments. The conclusion is that such an interpretation can stand, and moreover that it allows one to improve the physical content of the reaction description itself. In addition it forces one to define quite specifically the set of labels which is necessary and sufficient to characterize an incoming "TDHF channel" uniquely, and which has no more information than the TDHF theory can convey about the isolated individual nuclei in the distant asymptotic region. The result of this analysis is the conclusion that the TDHF reaction theory describes the scattering and reaction of certain mathematical objects which we refer to as "TDHF droplets." These objects are of a classical character, at least in the realm of small amplitude vibrations, having a discreet set of normal mode frequencies at which they can vibrate, but being allowed a continuous range of amplitude and 
phase for these (therefore classical) vibrations. One thus arrives at the intriguing situation where scattering and reaction process for classical objects is described in terms of a matrix element formed from two approximate (the TDHF time dependent quantum mechanical wave amplitudes.

Further effort is aimed at understanding and interpreting the differences between $\mathcal{S}$ and the exact S-matrix, especially the fact that conservation of probability unitarity is: not automatic in the 8 -TDHF approximation. (J.J. Griffin, P.C. Lichtner and M. Dworzecka).

C. Theoretical Commensurability in Complex Nuclear Theories

The principle of comnensurability has been formulated and proposed as a method to measure theories, especially theories involving complex multi faceted phenomena, such as the nuclear heavy ion reactions, The principle states the nearly tantological remark that a good theory ought to give results commensurable with its structure and with its input. The correlary that theories which give too much or require too little in the way of input are suspect either of error, or of hidden assumptions, is the practical tool for applying the principle of commensurability to complex theories. This approach to the critical analysis of theoretical adequacy has been applied to a preliminary way to some of the methods used to study nuclear heavy ion reactions (see item D. below). (J.J. Griffin).

\section{Survey of Theoretical Approaches to Heavy Ion Physics}

Guided by the principle of commensurability mentioned above, we have undertaken to tabulate the characteristics of nuclear systems, and the input requirements of various theoretical approaches to the nuclear 
heavy ion proble in order to measure the commensurability of the various theories with the physical content of the problem they attempt to describe: This process gives an interesting overview of the many ways in which people have set out to describe the multi-faceted aspects of these very complicated reaction processes. The survey at present is still in its preliminary stages, but even so it has enabled one to identify certain aspects of the nuclear physics which are not incorporated in some of the more popular theoretical descriptions.

On the other hand it has also exhibited the simple fact that a theory like TDHF does incorporate all the major qualitative features, in no way guarantees that that theory will give a good description of the nuclear data measured for the actual process. Indeed, the fact that a theory does successfully incorporate various qualitative features of ten goes with the fact that it has nevertheless, strenuously oversimplified those qualitative features, consistently, in order to make them manageable theoretically. Thus it is seen to be true from our survey, that the best phenomenological description of the data may come not from a thcory which is conceptually complete, but which in fact, to the contrary, is narrowly focused at the particular mechanisms and data which it attempts to describe quantitatively. On the other hand, a theory which includes in some way the fill1 range of conceptual structure exhibited by the underlying physical problem is likely to be so oversimplified (because of practical considerations) that its predictions are also simplistic, in spite of their superior conceptual relevance to the physics at hand. This is especially the case for the time dependent Hartree-Fock theory, - which therefore emerges as the system of special interest for theorists, rather than as a practical means 
to predict numerical comparisons adequate to the measured laboratory data. (J.J. Griffin, P.C. Lichtner and M. Dworzecka*).

E. Rayleigh Instability in the Colliding Water Droplets

This process has been analyzed in the quadratic approximation and the results have been reported as a thechnical report (ORO 4856-55). We believe that the Rayleigh instability is the correct physics for describing these "necklace events" in the break up of water droplets colliding with sma11 impact parameter, and that the quadratic approximation which we have used describes at least the higher water modes of this breakup process adequately. Further study on the role of odd-parity vibrations in the breakup process for collisions of unequal droplets is continuing. (J.J. Griffin and C.Y. Wong, O.R.N.L.).

\section{F. TDHF Solution as a Dynamical Basis}

Work on the use of the TDHF approximation as a dynamical basis with which to expand the many-body time dependent wave function is in the process of completion. Recent work has included a systematic procedure for correcting the TDHF Slater determinant by adding time dependent particle hole states as well as establishing a connection to the Goldstone linked cluster expansion for the stationary problem. (J.J. Griffin and P.C. Lichtner).

\section{G. Instant Particle Emission in Heavy Ion Collisions}

Recent experiments indicate the emission of particles during the initial stages ( $10^{-23} \mathrm{sec}$ ) of heavy-ion collision. An order of magnitude estimate shows that the kinetic energy of the emitted particles ( $20 \mathrm{MeV}$ ) is equal to

* Summer visitor from A.V. Humboldt Fellowship at Technische Hochschule, Darmstadt, W. Germany. 
the amount of surface energy released during neck formation. Initial calculations for the probability of emission using a realistic two-center Woods-Saxon shell model in a sudden approximation indicate that two-body effects are most likely to dominate: (J.J. Griffin and P.C. Lichtner).

\section{H. Application of the Window Formula to the Grazing Heavy-ion Collisions}

The coupling of "slow" collective motion of a large nuclear system to the "fast" single particle degrees of freedom was treated by means of the one body dissipation mechanism-window formula and applied to the macroscopic description of the grazing heavy ion collisions.

The explicitly treated coupled collective variables were; the separation of the c.m.: of the approaching nuclei, and the three angular momentarelative and intrinsic. The motion of the system was governed by RaleighLagrange force obtained from Coulomb, surface and proving potential energies. The frictional, dissipative force was obtained from window formula. With no adjustable parameters we. were able to obtain the angular deflection and energy losses in the grazing collisions, for selected reactions, which are in a very good agreement with the experimental data. (M. Dworzecka*, .T. R1,ock.i ${ }^{\dagger}$ and $F_{\mathrm{a}}$ Beck $^{\dagger}$ ).

\section{Semiclassical Approximation to Time-Dependent Hartree-Fock Theory}

Within a Time-Dependent Hartree-Fock framework, we have deyeloped a semiclassical approximation appropriate for large systems,

This approximation scheme is obtained by transforming the Time-Depend-. ent Hartree-Fock equations to the phase space via Wigner distribution function. The kinetic equation for the distribution function can be

* 1977 Summer visitor from A. v. Humboldt Fellowship at Technische Hochschule, Darmstadt, W. Germany.

$\dagger$ Technische Hochschule, Darmstadt, W. Germany. 
simplified, if one assumes that $h \bar{\nabla}_{r} \cdot \bar{\nabla}_{k}$ is a valid expansion parameter. We have shown, that the termination of the expansion at any order preserves the conservation of the energy and the number of particles for the system.

We demonstrate that the standard semiclassical approach, the ThomasFermi approximation, is inconsistent with Hartree-Fock.theory when the basic two-body interaction is short-ranged (as in nuclear systems, for example). However, by introducing a simple extension of the ThomasFermi approximation, we overcome this problem, We also discuss the infinite nuclear matter problem and point out that Time-Dependent HartreeFock theory yields collective modes of the zero sound.variety instead of ordinary hydrodynamic (first) sound, We thus emphasize that one should be extremely circumspect when attempting to cast the equations of motion of Time-Dependent Hartree-Fock theory into a hydrodynamic-like form. (M. Dworzecka* and R. Poggioli),

J. Semiclassical Approximations for a Momentum Dependent One-Body Potential

We have generalized the semiclassical method Jennings, et al:, a method to calculate shell corrections alternative to Strutinsky's method, to a spherical but a momentum dependent potential of the form, $V(r)+\frac{1}{2}$ $\left(p^{2} W(r)+W(r) p^{2}\right)$. Explicit expressions are developed for the number of particles and the smooth sum of single particle energies in terms of the Fermi energy and the one-body potential and its first two derivatives. They are calculated for selected values of the parameters and compared with the sum of single particle energies obtained by numerical solution of the

1977 summer visitor from A.v. Humboldt Fellowship at Tech, Hochschule, Darmstadt, $W$, Germany. 
Schrbdinger equation. The difference between the two is essentially the shell correction. The shell correction can be roughly estimated using a simple isotropic harmonic oscillator model. It is also pointed out that the semiclassical energy is not the same as the energy averaged over a major oscillator shell. (M. Dworzecka* and S. Moszkowski ${ }^{\dagger}$ ).

\section{K. Shells and Clusters in Deformed Nurlei.}

Shell effects in deformed (e.g. fissioning) nuclei and their relation. to the formation of substructures has been studied in terms of a semiphenomenological resonating group representation. In this approach the shell energy is attributed to the microscopic inter structure whereas the major part of the total energy is replaced by the macroscopic liquid-drop energy. Thus nuclear deformations are related to cluster amplitudes. In this way the occurence of so-called "magic" deformations can be interpreted as the formation of spherical doubly-magic substructures. In particular the ground-state and the reflection-asymetric isometric minimum in the actinides can be explained by an energetically favored formation of $(Z=82, N=126)$ and $(Z=50, N=82)$ substructures, respectively, An analysis of the shell energy in terms of there compound neutrons numbers $\mathrm{N}=132$ together with the $(z=50$ substructure determines the border between symmetric and asymmetric fission in the radium region. For very heavy actinides a comparison of the relevant two-cluster terms in this expansion indicates that in all Fermium isotopes the symmetric fission mode is enhancedby $(Z=50)$ substructures, whereas, it is a pure liquid-drop effect in all lighter actinides, (H, Schultheis ${ }^{\dagger+}, R$. Schultheis, F. Glnnenwein, K. Wildermuth (U. Tlbingen, W. Germany).

* 1977 summer visitor from A.v. Humboldt Fellowship at Technische Hochschule Darmstadt, W. Germany

$\dagger \quad 1975$ visitor from UCLA

+ 1977-78 visitor from U. of Ttbingen, W. Germany 
L. Alpharparticle Model Calculation in ${ }^{32} \mathrm{~s}$ as a Microscopic Model for Fission

The formation and break-up of substructures as studied in ${ }^{32} \mathrm{~s}$ as a calculable microscopic model for analogous long-range many-nucleon correlations in fission of actinides. The calculations are performed for alphacluster wave functions with Volkov and Brink-Boeker interaction allowing for $\alpha,{ }^{12} \mathrm{C},{ }^{16} \mathrm{O}$ and ${ }^{20} \mathrm{Ne}$ clustering. We find two separate valleys in the potential energy landscape that are associated with different types of correlations in the wave function and two local energy minima that are analogous to the ground state and isomeric state of actinide nuclei: The minimum at larger deformation (i.e. larger quadrupole moment and larger rms radius) is associated with an ${ }^{16} 0-{ }^{16} 0$ "fragment" substructure whereas no such substructure is found in the ground state minimum of ${ }^{32} \mathrm{~S}$. It turns out that the correlated motion of large magic numbers of nucleons in two groups $\left({ }^{16} 0+{ }^{16} 0\right)$ is energetically favourable already at relatively small deformations: In the second minimum the ${ }^{16} 0+{ }^{16} 0$ substructure occurs with high probability (about $80 \%$ ). In analogy to these results the "pre-formation" of fragments and "fragment she11" effects occuring in the fission of actinides are explained in terms of probability statements for the formation of the corresponding heavy clusters in the many-body wave function. (H. Schultheis*, R. Schultheis*, K. Wildermuth (U. Ttbingen, W. Germany); A. Faessler, F. Grummer (K.F.A. JUlich, W. Germany)).

M. Energy Dissipation in the Fission of ${ }^{252} \mathrm{Cf}(\mathrm{sf})$

The problem of energy dissipation in the fission of heavy nuclei has been studied by a number of groups in various approaches with contradic-

* 1977-78 visitur from U. of Tubingen, W. Germany. 
tory results. Estimates of the dissipated energy have been obtained from experimental information, from hydrodynamical calculations, from particular microscopic models or from combinations of several of these approaches. We have studied the amount of energy that is available for dissipation by relating the potential energy of the fissioning nucleus to the experimental post-fission data: Numerical results have been determined for the spontaneous fission of ${ }^{252}$ Cf by taking into account a two-spheroid scission configuration with diffuse surface, nuclear interaction and Coulomb excitation effects, The results indicate low dissipation. "The internal plus non-translational collective excitation energy at scission 1 s found to be at most $8 \mathrm{MeV}$. We have also studied the range of fragment deformations at scission that are compatible with the data. It turned out that the fragment deformation at scission is determined by the shell effect associated with the ground state deformation of the heavy fragment rather than by the conventional hypothesis of minimum potential energy at scission. (H. Schultheis* and R. Schultheis*).

\section{N. Predictions of Ternary Fission Rates for Element 126}

Motivated by the reported cxperimental evidence for super heavy elements in giant halo formations in certain micas, we have calculated the ternary and binary fission barriers and their relative WKB penetrability for the isotopes $184 \mathrm{~s} \mathrm{~N} s 228$ of element 126, considering both $\mathrm{Z}=114$ or $\mathrm{g}=126$ as magic numbers. In all cases the predicted relative rates $r_{T} / r_{B}$ of ternary $(T)$ to hinary (B) low energy fission events turned out to be substantially higher than those known for actinides. We have therefore proposed to measure the ternary fission events. from the samples as a test for super heavy element 126.

* 1977-78 visitor from U, of Tubingen, W, Germany. 
However, the experimental evidence became less strigent during the process of publication of our proposal. (H. Schultheis* and R, Schultheis*).

o. An Algorithm for Non-Smoothing Contour Representations of Two-Dimensional Arrays

A FORTRAN Code has been developed, which calculates, plots and labels approximate contour lines $\mathrm{Z}(\mathrm{x}, \mathrm{y})=\mathrm{C}$ for data that are given as a rectangular array $z_{i k}=z\left(x_{i}, y_{k}\right)$. The algorithm interpolates roots along the meshlines and approximates the contour line by straignt lines within each grid cell. Our algorithm has been developed for the study of local energy minima and saddle points in nuclear fission theory. Here the precise assignment of contour lines to grid cells is essential whereas the degree of accuracy achieved within each grid cell is unimportant. Therefore, smooth contour plotting methods that envolve derivatives; small step sizes or curve fitting procedures are less appropriate.

The correct correspondence between curve and cell is particularly important for contours with degeneracies. Therefore, our algorithm treats the problems of degenerate cells and degenerate nodes in a proper way. Degenerate nodcs arc trcated without approximations hy taking also the corners of each grid cell into account (cf. fig. 1). Degenerate cells are isolated, and marked by multiple connections if the contour line cannot be traced on the basis of the input data. The conventional methods allow for failure in such cases or bypass the problems by adding arbitrary 'corrections' to the input data and making assumptions about the local behavior of neighboring curves. Then the degeneracies do not occur in the final plot. ( H. Schultheis* and R. Schultheis*).

* 1977-78 visitor from U. of Tubingen, W. Germany. 


\section{APPENDIX}

A. Research Publications - March: $1 ; 1976$-August $31 ; 1977$

GAUGE TRANSFORMATIONS AND QUANTUM MECHANICS (I): GAUGE INVARIANT INTERPRETATION: OF QUANTUM MECHANICS, K. H. Yang, Ann. Phys. (N.Y.) 101 (19.76),62,

GUAGE TRANSFORMATIONS AND QUANTUM MECHANICS (II): PHYSICAL INTERPRETATION: OF ClASSICAL GAUGE TRANSFORMATIONS, R. H. Yang, Ann. Phys. (N.Y:) I01, (1976), 97.

MULTIPLE SCATTERING AND NUCLEON RECOIL EFFECTS IN PION PRODUCTION BY NUCLEONS ON NUCLEI, K. H. Yang, D. A. Sparrow, B. R. Holstein and M. M. Sternheim, Phys. Rev. C14, (1976), 1083.

NON-EIKONAL APPROACH TO SCATTERING OF HADRONS FROM NUCLEI: II. PROTON AND PION SCATTERING FROM $D,{ }^{3} \mathrm{He},{ }^{4} \mathrm{He}\left(k_{\mathrm{L}} 1 \mathrm{GeV} / \mathrm{c}\right), \mathrm{Y}$.-A.jexander, S...A...Gurvitz, and A. Rinat, Ann. Phys. (N.Y.) 28 (1976) 322

INVARIANT POTENTIAL FOR ELASTIC PION-NUCLEUS SCATTERING, M. K. Banerjee, and I. B. Cammarata, Phys. Rev. C13 (1976),299.

COLLIDING HEAVY IONS: NUCLEI AS DYNAMICAL FLUIDS, J J.' Griffin and K. K, Kan, Rev. Mod. Phys. 48 (1976) 467.

EVOLUTION OF A QUANTUM SYSTEM: LIFETIME OF A DETERMINANT, J. J. Griffin and P. C. Lichtner, Phys. Rev. Lett. 37 (1976)1521..

OPEN QUESTIONS ON NUMERICAL TIME-DEPENDENT HARTREE-FOCK MODEL, J: $:$ J. Griffin and P. C. Lichtner, U. of Md. Tech. Rept. 76-096 (April 1976).

VIBRATIONAL INSTABILITY: A POSSIBLE TEST OF NUCLEAR HYDRODYNAMICS, J. J. Griffin and C. Y. Wong, U. of Md. Tech. Rept. 非6-118 (June 1976).

LOW ENERGY PION NUCLEON SCATTERING, M. K. Banerjee, and J. B. Cammarata, (to appear in Phys. Rev. D, Sept. 1, 1977)

THFORY OF LOW ENERGY PION NUCLEON SCATTERING, M. K. Banerjee and J. B. Cammarata U. of Md., Tech. Rept.非7-156 (submitted to Phys. Rev. C)

PION NUCLEON o COMUTATOR, M. K. Banerjee and J. B. Cammatata, Univ. of Md. Tech. Rept. $\$$ 177-277 (submitted to Phys. Rev, D)

A SEMI-CLASSICAL APPROXIMATION OF TIME-DEPENDENT HARTREE-FOCK THEORY, M. Dworzecka, and R. Poggioli, U, of Md. Tech. Rept. 非6-115

A NEW APPROACH TO ANTISYMMETRIZATION AND REARRANGEMENT IN NUCLEUS-NUCLEUS COLlisions, P. C.Lichtner and K. Pruess, Nucl. Phys: A269 (1976) 252 .

CALCULATIONS OF SINGLE PARTICLE POLARIZATION USING A REALISTIC TWO-CENTER SHELL MODEL, P. C. Lichtner and K. Pruess (to be published).

CORRELATIONS AND DIPOLE EXCITATIONS IN NUCLEI, W. M. MacDonald, and A. Mekjian, Phys. Rev. C15 (1977) 531.

LOCALIZATION IN HEAVY ION ELASTIC, INELASTIC AND TRANSFER REACTIONS, P. J. Moffa, C. B. Dover and J. P. Vary, Phys. Rev. C13(1976)147. 
DECOMPOSITION OF THE SMOOTH CUT-OFF MODEL ANGULAR DISTIRBUTION, P. J. MOffa, and R. C. Fuller, Phys. Rev. C14 (1976)1721.

NUCLEAR AND COULOMB COMPONENTS IN HEAVY ION ELASTIC AND TRANSFER REACTIONS P. J. Moffa and R. C.Fuller, Phys. Rev. C15 (1977) 266.

A MICROSCORIC ION-ION SPIN-ORBTT POTENTIAL, P. J. Moffa, U. of Md. Tech. Rept. 非7-080 (to be published in Phys: Rev. C.)

A FOLDING MODEL DESCRIPTION OF HEAVY ION INELASTIC SCATTERING P. J. Moffa, C. B. Dover and J. P. Vary, BNL Rept. 非2431 (to be published in Phys. Rey. C)

A HEURISTIC METHOD FOR DETERMINING OUTGOING WAVES IN MANY-BODY WAVE FUNCTIONS, E. F. Redish, P. C. Tandy \& M. L'Huillier, Phys. Lett. 61B (1976) 413.

A THREE-BODY APPROACH TO THE SINGLE SCATTERING OPTICAL POTENTIAL, E. F. Redish P. C. Tandy and D. Bolle', U. of Md., Tech. Rept. \#⿰⿰77-058(submitted to Phys. Rev.)

A NEW PROOF OF THE BENCZE-REDISH-SLOAN EQUATIONS, E, F. Redis, M. L'Huillier, P. C. Tandy, and B. Benoist-Gueutal, (submitted to Phys, Rev.)

LECTURES IN THE QUANTUM THREE-BODY PROBLEM, E. F. Redish, U. of Md. Tech. Rept. 非7-060, February 1977.

THE STRUCTURE OF THE MANY-BODY WAVE FUNCTION FOR SCATTERING, E. F. Redish, P. C. Tandy and M. H'Huiliier, Univ. of Md., Tech, Rept. \#18-003, July, 1977.

NUCLEON KNOCK-OUT WITH KAONS, E. F. Redish, P. J. Moffa and R. D, Koshel, Univ, of Md. Tech. Rept. 非8-011, August, 1977.

CLUSTER ASPECTS OF NUCLEAR FISSION, H. Schultheis, G. Gönnenwein, R. Schultheis and K. Wildermuth, Z. Physik A278 (1976)15.

PREDICTION OF TERNARY FISSION RATES FOR ELEMENT 1.26, H. Schultheis, R. Schultheis, Phys. Rev: C15 (1977)1601.

ALGORITIM FOR NON.SMOOTHING CONTOUR RFPRFSFNTATIONS OF TWO-DIMENSIONAL ARRAYS, H. Schultheis, and R. Schultheis, submitted to the Computing $J$.

SOME BOUNDS FOR ENERGY DISSIPATION AND FRAGMENT DEFORMATION IN ${ }^{252} \mathrm{Cf}$ (sf), H. Schultheis and R. Schultheis, submitted to Nucl, Phys. A.

A STUDY OF HIGH SPIN STATES IN ${ }^{151} \mathrm{Gd}$, A. B. Volkov, H. J. Smith M. W. Johns, G. Lovhoiden, J. V. Thompson, and J. C. Waddington, Can. J. Phys: 55, (1977) $690-713$.

COMPARISON OF EXACT AND APPROXTMATE MULTIPLE SCATTERING .CALCULATIONS, S, J, Wallace, Y. Alexander and D. A. Sparrow, Nuc1. Phys. A280 (1977), pp.285-307. ELASTIC $\mathrm{p}^{-4} \mathrm{He}$ SCATTERING NEAR $1 \mathrm{GeV}, \mathrm{S}$. J. Wallace and Y. Alexander, Phys. Rev, Lett. 38 (1977), 1269.

RELATIVISTIC EIKONAL EXPANSION, S, J. Wallace and J. McNeil, Univ, of Md. 'Tech. Rept. \#78-009, (August 1977).

GAUGE TRANSFORMATIONS AND QUANTUM MECHANICS (III) : THE CONVENTIONAL INTERPRETATION OF QUANTUM MECHANICS, R. H. Yang, Univ, of Md. Tech, Rept. 非7-019, August 1976. 
GAOGE INVARIANCE OF THE RAYLEIGH-SCHRODINGER TIME-DEPENDENT PERTURBATION THEORY, K. H. Yang, Univ, of Md., Tech. Rept, 非8-012, August, 1977.

POYNTING'THEOREM AND ELECTRONS WITH SPIN, K. H. Yang, UnIV, of Md., Tech. Rept, 非78-013, August, 1977.

PARTIAL WAVE EXPANSION FOR ARBITRARY SPIN AND THE ROLE OF NON-CENTRAL FORCES, R. C. Johnson, Univ. of Md. Tech. Rept. 非77-023 (submitted to Nucl. Phys.).

DEUTERON BREAK-UP EFFECTS IN (p,d) REACTIONS AT $65 \mathrm{MeV}, \mathrm{R}, \mathrm{C}$. Johnson and G. L. Wales, Nucl. Phys: A274-(1976) 168-176.

BOUND STATE MOMENTUM DISTIRBUTION, Y. Alexander, N.S. Wall, and E. F. Redish, Univ. of Md. Tech. Rept. \#17-048 (to be published in Phys. Rev, C).

A SEMI-CLASSICAI APPROXIMATION FOR A MOMENTUM DEPENDENT ONE-BODY POTENTIAL, M. Dworzecka and S. Moszkowsk1; Univ. of Md. Tech. Rept. 77-021 (submitted to Phys. Rev. 2

RECOIL AND STRONG FORM FACTOR EFFECTS IN MESONIC EXCHANGE CORRECTIONS ON THE.. CHARGE DISTRIBUTION OF ${ }^{3} \mathrm{He}$ AND ${ }^{3} \mathrm{H}, \mathrm{W}, \mathrm{M}$, Kloet and $\mathrm{J}$, A, Tjon, Phys. Lett $61 \mathrm{~B}$ (1976) . P. 356.

ANALYSIS OF ELASTIC AND INELASTIC $-{ }^{12} \mathrm{C}$ DATA $(E=1.37$ GeV)..WITH A MULTIPLE DIFFRACTION MODEL. Y. Alexander and A, Rinat, Nucl, Phys, A278 (1977) 525-532, 
B. Contributed Papers March 1, 1976 - August 31, 1977 :

SEMICLASSICAL APPROXIMATION IN TIME-DEPENDENT HARTREE-FOCK, M. Dworzecka and R. Poggioli, Bull. Am. Phys. Soc: 21, 12 (1976),

SCATTERING WAVE FUNCTIONS IN THE N-BODY PROBLEM, E. F. Redish, M. L'Huillier and P. C. Tandy, Bull. Am. Phys. Soc. 21, 49 (1976).

GAUGE-INVARIANT INTERPRETATION OF QUANTUM MECHANICS, H.-H. Yang, Bull, Am. Phys. Soc. 21, 23 (1976).

HADRON NUCLEUS SCATTERING IN A NON-EIKONAL APPROACH, S, A, Gurvitz, and Y. Alexander, Bull. Am. Phys, Soc: 21, 519 (1976),

SEMICLASSICAL APPROXIMATIONS FOR A MOMENTUM DEPENDENT ONE-BODY POTENTIAL, M. Dworzecka and S.A. Moszkowsk1, Bull. Am. Phys. Soc, $\underline{21}, 614$ (1976).

CORRELATIONS AND DIPOLE EXCITATIONS IN NUCLEI, A. Z. Mekjian and W. M. MacDonald, Bul1, Am, Rhys, Soc, 21, 560 (1976).

THE PAULI PRINCIPLE AND THE SPIN-DEPENDENCE OF THE DEUTERON-NUCLEUS INTERACTION, R. C. Johnson and A, A, Ioanides, Bull, Am, Phys, Soc: 21, 539 (1976).

INFLUENCE OF AN ISOSPIN DEPENDENT NUCLEON-NUCLEUS SPIN-ORBIT POTENTIAL IN ANALYZING POWERS IN (d,p) REACTIONS, R. C, Johnson and G. L. Wales, Bu11. Am, Phys, Soc, 21, 540 (1976).

THE (p,d) REACTIONS AT $65 \mathrm{MeV}, \mathrm{R}, \mathrm{C}$. Johnson and G. L. Wales, Bu11. Am. Phys. Soc. 21, 540 (1976).

DO WE REALLY KNOW GAUGE TRANSFORMATIONS OF THE SECOND KINE?, Kuo-Ho Yang, Bul1. Am. Phys. Soc, 21, 596 (1976).

ONE EXAMPLE OF THE GAUGE DEPENDENCE OF "THE USUAL INTERPRETATION OF PROBABILITY AMPLITUDES," Kuo-Ho Yang, Bu11. Am. Phys. Soc. 21, 596 (1976).

PION NUCLEUS ELASTIC DIFFERENTIAL SCATTERING AT LOW ENERGY, M, K. Banerjee and S, J, Wallace, Bull, Am. Mhys. Soc, '21, 984 (19\%6),

INELASTIC PION SCATTERING FROM LIGHT NUCLEI, D. A. Sparrow, Bull, Am. Phys. Soc. 21,518 (1976).

EXACT FINITE RANGE CALCULATIONS OF ( $p, d)$ REACTION CROSS SECTIONS AT $\mathrm{E}_{\mathrm{p}}=185$, 360, 540, AND $700 \mathrm{MeV}, \mathrm{D}, \mathrm{A}$, Sparrow, Bull. Am. Phys, Soc. 21, 519 (1976).

ANALYSIS OF THE ${ }^{4} \mathrm{He}(\mathrm{p}, \mathrm{d})^{3} \mathrm{He}$ REACTION AT $770 \mathrm{MeV}, \mathrm{D}$. A. Sparrow, Bull. Am. Fhys, Soc, $21,964(1976)$.

HIGH-ENERGY EXACT FINITE RANGE DWBA CALCULATIONS FOR ( $p, d)$ REACTIONS ON ${ }^{58} \mathrm{Ni}$, $90 \mathrm{Zr}$, and $208 \mathrm{~Pb}, \mathrm{D}$. A, Sparrow, Bull, Am. Phys, Soc: 21,964 (1976).

BOUND STATE MOMENTUM DISTRIBUTION, Y, Alexänder, E, F, Redish and N, S, Wall, Bul1. Am. Phys, Soc, 21, 971. (1976). 
MODEL THREE-BODY PROBLEM, A, Fonseca, Bull, Am. Phys. Soc. 21 (1976),

TEST OF TRUNCATION IN A FOUR-BODY PROBLEM, A. Fonseca, and E. Fil Redish, BuIl. Am. Phys, Soc. 22, 530 (1977).

"INSTANT DISSIPATION" IN HEAVY ION COLLISION, J. J. Griffin and D. Gross, Bull. Am. Phys. Soc. 21, 973. (1976).

PROJECTILE EXCITATION IN A FOLding MODEL, P. J, Moffa, C, B. Dover and J, P. Vary, Bull. Am. Phys. Soc. 21, 975 (1976).

A NEW VIEW OF BREAKUP EFFECTS IN DEUTERON STRIPPING REACTIONS, E, F, Redish, Bul1. Am, Phys. Soc. 21, 964 (1976).

CALCULATIONS OF SINGLE PARTICLE POLARIZATION USING A RELATIVISTIC TWO-CENTER SHELL MODEL, P. C, Lichtner and K, Pruess, Meeting on Heavy-Ion Collisions, Falls Creek Falls State Park, Tennessee (June 1977).

USING LOCAL MOMENTUM TO DISENTANGLE ANGULAR DISTRIBUTIONS, P. J, Moffa and R. C. Fuller, Proc, of the Symposium of Macroscopic Features of Heavy Ian Collisions, Argonne, Illinois (1976), p. 575,

MONOPOLE EXCITATIONS INDUCED BY PIONS, D, A, Sparrow, Bull, Am, Phys. Soc. 22, 80 (197.7).

PRO'ION HELIUM ELASTIC SCATTERING, Y. Alexander and S. J. Wallace, Bull, Am. Phys. Soc, 22,561 (1977),

RIGID BODY MOMENTS AND MANY-INDEPENDENT PARTICLE SYSTEMS, J. J, Griffin and K, -K, Kan, Bul1. Am. Phys. Soc, 22, 529 (1977).

CALCULATION OF THE $\left(\mathrm{K}^{+}, \mathrm{K}^{+} \mathrm{p}\right)$.REACtion, P. J. Moffa, R. D. Koshel and E. F. Redish, Bu11. Am. Phys. Soc. 22, 617 (1977).

CONVERGENCE OF THE DWBA SERIES, E. F. Redish, and D. S. MacMillan, Bull. Am. Phys. Soc. 22, 598 (1977).

THE RELATIONSHIP OF CONNECTED KERNEL THEORY TO THE SCHRÖDINGER EQUATION AND IMPLICATIONS FOR REACTION MODELS, E. F. Redish, P. D. Tandy, Gy, Bencze, and M. L'Huillier, Internat'1. Symp. on Nuclear Reaction Models, Balantonfüred, Hungary (June 27-July 1, 1977).

RELATIVISTIC EIKONAL EXPANSION, J. McNeil and S. J. Wallace, Bu11, Am. Phys. Soc, 22, 615 (1977).

THREE-BODY MODEL OF HEAVY ION REACTIONS, P. E. Shanley and A. C. Fonseca, Bul1. Am, Phys, Soc, 22, 530 (1977):

MODEL THREE-BODY PROBLEM," A, C, Fonseca, Bull. Am, Phys, Soc. 22, 530 (1977), 
$\mathrm{N}^{*}$ Virtual production in p-nUCleus elastic scattering, S. J. Wallace, Buil. Am. Phys. Soc. 22, 561 (1977).

CHARGE DEPENDENCE OF NUCLEON-NUCLEON SCATTERING LENGTHS AND THE PION-NUCLEON COUPLING CONSTANTS, T. B. Wells, Bull. Am. Phys. Soc. 22, 616 (1977).

ELASTIC $\mathrm{p}-{ }^{4} \mathrm{He}$ SCATTERING NEAR $1 \mathrm{GeV}, \mathrm{S}$. J. Wallace and Y. Alexander, VII Internat'l. Conf. on High Energy Physics and Nuclear Structure, Zurich, Switzerland (Aug. 29-Sept. 2, 1977). 
C. Invited Talks - March $1 ; 1976$ - August $31 ; 1277$

THE SPIN-DEPENDENCE OF THE DEUTERON-NUCLEUS INTERACTION, $R, C$. Johnson, Bull. Am. Phys, Soc: 21 , No. 4, P.654, Washington, D. C. Apri1, 1976.

FIELD THEORETIC ASPECTS OF PION NUCLEUS PHYSICS, M. K. Banerjee, MesonNuclear Physics Conference, Carnegie-Mellon University, Philadelphia, Pa., AIP Conf. Proc. 333 , p. 119, May, 1976.

NUCLEON KNOCKOUT: REACTIONS MECHANISMS, E. F. Redish, Conference on Determination of Momentum Space Wave Functions in Atoms, Molecules, and Nuclei, Bloomington, Indiana, June, 1976, AIP Con̈f. Proc. 1977.

THEORETICAL COMMENSURABILITY AND THE TIME-DEPENDENT HARTREE-FOCK CAICULATION, J. J. Griffin, 1976 Canadian Theoretical...Physics Conference (Kingston, Ontario, Canada, September, 1976).

APPLICATIONS OF THREE-BODY METHODS TO NUCLEAR PROBLEMS, E. F. Redish, European Summer Meeting on Few Body Nuclear Physics, Uppsala, Sweden, June 17-19, 1977.

NUCLEAR HEAVY ION PHYSICS, J. J. Griffin, Fifth Jubilee Conf, of the Moscow. Seminar on Collective Phenomena (April 17-20, 1977, Moscow, USSR).

NUCLEAR FERMI DYNAMICS: PHYSICAL CONTENT VS. THEORETICAL APRROACH, J. J. Griffin, Conference on Heavy Ion Collisions, Fall Creek Falls, Tenn June $13-17,1977$ (E. Halbert ORNL ed.).

STRUCTURE OF THE SCATTERING WAVE FUNCTION IN THE M-BODY PROBLEM, E. F. Redish, International Symposium on Nuclear Reaction Models, Balatonfured, Hungary, June 27-July 1., 197.7.

MOLECULAR STATES IN THE NUCLEAR THREE-BODY PROBLEM, Aritonio Fonseca, International Symposium on Nuclear Reactions, Balatonfured, Hungary, June 27-July $1,1977$.

SOLUBLE MODEL OF THE FOUR-NUCLEON SYSTEM, Antonio Fonseca, International Symposium on Nuclear Reactions, Balatonfured, Hungard, June 27-July 1, 1977.

GENERALIZED FADDEEV APPROACH TO MANY-BODY REACTIONS, E. F. Redish, Gordon Research Conf. on Few Body Probems in Chemistry and Physics, New Hampshire, August 15-19, 1977.

NUCLEAR FERMI DIAMMICS, J. J. Criffin, The Internatinnal Symposium on Nuclear Collisions and their Microscopic Description, M. Mihailovic, ed. (September 26-0ctober 1, 1977, Bled, Yugoslavial.

SURVEY OF THEORETICÄL MODELS FOR CLOSE HEAVY ION COLLISIONS, J, J, Griffin APS Dïvision of Nuclear Physics Meeting (Rochester, N, Y.:Ocotber 27-29,1977). 


\section{Papers in Preparation}

COMPARISON OF THE NON-EIKONAL APPROACH .AND CORRECTIONS TO THE EIKONAL MODEL TO AN EXACT CALCULATION, Y. Alexander, S. J. Wallace and D. Sparrow,

QUASI-FREE KAON SCATTERING, Y, Alexander and P. J. Moffa.

CORRELATIONS IN ${ }^{4} \mathrm{He}, \mathrm{Y}$. Alexander and $\mathrm{S}, \mathrm{J}$. Wallace,

QUASI-ELASTIC $\mathrm{N}^{*}$ PRODUCTION, Y. Alexander. E. F. Redish and S, J. Wallace.

APPLICATION OF THE WINDOW FORMULA TO THE GRAZING HEAVY IONS COLLISIONS,

M. Dworzecka, J. Blocki and F. Beck.

THE $\&$-MATRIX FORMULATION OF THE TDHF REACTION THEORY, M, Dworzecka, J, J. Griffin and P. Lichtner.

CRITIQUE OF THE TIME-DEPENDENT HARTREE-FOCK REACTION THEORY, M. Dworzecka, J. J. Griffin and P. Lichtner.

ROTATIONAL MOTION FROM THE SINGLE PARTICLE SCHRÖDINGER FLUID VIEWPOINT, J. J. Griffin and K.-K. Kan.

VIBRATIONAL INSTABILITY IN COLLIDING WATER DROPLETS, J..J, Griffin and C. Y. Wong.

A DYNAMICAL BASIS FROM TDHF, J. J. Griffin, P. C. Lichtner, $\cdot K_{1}-K_{\text {, Kan }}$ and T. Tamura.

TIME-DEPENDENT HARTREE-FOCK APPROXIMATION AND ITS APPLICATION TO HEAVY-ION SCATTERING, P. C. Lichtner, M. Dworzecka, and J. J. Griffin.

ENHANCEMENT OF THE EFFECT OF 2p-2h CORRELATIONS IN DIPOLE SUM RULES, $W$. $M$. MacDonald and A. Mekjian.

CALCULATIONS OF THE $\left(\mathrm{K}^{+}, \mathrm{K}^{+} \mathrm{p}\right)$ REACTION, P. J. Moffa, R. D. Koshel and E. F. Redish.

A SIMPLE GRAPHICAI DISPLAY PROGRAM FOR PHYSICS, P. J. Moffa.

ASYMPTOTIC PROPERTIES OF GENERALIZED FADDEEV WAVE FUNCTIONS, E. F. Redish, P. C. Tandy and M. L'Huillier.

NON-LOCALITY STRUCTURE OF THE FIRST ORDER OPTICAL POTENTIAL, E, F. Redish.

STUDY OF MANY-NUCLEON CORRELATIONS IN ${ }^{32}$ S AS A MODEL FOR FRAGMENT SHELL EFFECTS IN FISSION, H, Schultheis, R, Schultheis, K. Wildermuth, A. Faessler and $\mathrm{F}$, Grummer.

HIGH SPIN STATES IN EVEN-EVEN NUCLEI, A, Volkov,

HIGH SPIN STATES IN ODD-EVEN NUCLEI, A. Volkov. 
$\mathrm{p}-{ }^{4}$ He ELASTIC SCATTERING AT $1 \mathrm{GeV}, \mathrm{S}$. J. Wallace.

$\triangle$ VIRTUAL PRODUCTION PROCESS IN p-NUCLEUS SCATTERING, S. J. Wallace.

REIAATIVISTIC EIKONAL EXPANSION: FERMIONS, S. J. Wallace.

SPIN DEPENDENCE OF HIGH ENERGY AMPLITUDES, S. J. Wallace.

PION NUCLEUS ELASTIC SCATTERING, S. J. Wallace. 